\title{
LOS PROGRAMAS DE GARANTÍA SOCIAL ESPECIAL COMO RESPUESTA A NECESIDADES EDUCATIVAS ESPECIALES: Reflexiones sobre la realidad educativa en un Instituto de Educación Secundaria'
}

\author{
María Pilar Salas Franco \\ Investigadora Agregada del IER
}

\begin{abstract}
RESUMEN: Este trabajo nos ofrece una interesante reflexión sobre la necesaria respuesta educativa a la diversidad del alumnado que en este siglo XXI va a ser la característica fundamental de la práctica docente en las aulas. A través de este estudio conoceremos la experiencia llevada a cabo durante los últimos diez años en el Instituto Duques de Nájera de Logroño (La Rioja), en torno a los Programas de Garantía Social Especial como una acertada respuesta educativa para alumnos con deficiencia mental. Podremos constatar también en las páginas que siguen, cómo la esencia de la educación radica en la persona a la que va dirigida y no tanto en los contenidos que se imparten, recogiendo muy claramente el siguiente proceso: Primero, ¿quién aprende? Segundo, ¿Cómo aprende? Tercero, ¿Qué le interesa? Teniendo claros estos planteamientos, lo demás sólo es un complemento del que nos servimos para conseguir el fin último de la educación: el desarrollo de las potencialidades de cada persona.
\end{abstract}

ABSTRACT: This work offers an interesting reflection on the necessary educational answer to the pupil's diversity, which will be the most outstanding feature of the teaching practice in the classrooms. Thanks to this study, we will know the experience that has been carried out for the last ten years in the Duques de Nájera Institute of Secondary Education in Logroño (La Rioja), around the Social Special Guarantee Programmes, as a right teaching answer for pupils with mental deficiencies. We can also check in the next pages, how the main purpose of the Education consists in the person to whom it aims, rather than in the given subject; paying attention to this process: first of all "Who is the

1. Este trabajo se enmarca dentro de la investigación realizada para el curso de Doctorado del Departamento de Ciencias Humanas y Sociales de la Universidad de La Rioja "Respuesta educativa a la diversidad" realizado en el primer cuatrimestre de 2005. 
learner?", second "How does he/she learn?", and third "What are his/her interests?". Making this approach clearly, the rest is only a helpful implement we use to reach the furthest purpose of the Education: the development of each one's possibilities.

DESCRIPTORES: diversidad educativa, alumnos con deficiencia mental, Programas de Garantía Social Especial, formar y educar en el siglo XXI.

KEYWORDS: Teaching diversity, pupils with mental deficiencies, Social Special Guarantee Programmes, to train and educate in the XXIst century.

\section{INTRODUCCIÓN}

La propuesta que se plantea trata de recoger una reflexión sobre la práctica diaria que desde hace ya diez años se viene realizando en el IES Duques de Nájera de Logroño, donde desempeño tareas administrativas desde 1987, en aras de conseguir una formación profesional e integración de unos alumnos con necesidades educativas especiales, en su mayoría ligadas a deficiencias mentales. En las siguientes páginas se ofrece por tanto una visión personal sobre la experiencia docente, contemplada desde un ámbito ajeno a la propia práctica educativa pero que participa también en la formación de este alumnado colaborando en actividades propuestas por su profesorado a fin de conseguir una mayor integración en el Centro. Para ello es fundamental el apoyo ofrecido a estos alumnos para que puedan resolver situaciones cotidianas en las diferentes dependencias del Instituto, que en el caso concreto de su relación con la secretaría del mismo consisten en realizar tareas como: enviar o recibir un fax, hacer fotocopias, pedir material de oficina, plastificar carnets del alumnado o encuadernar listados de matrícula, horarios del profesorado, etc.

Así pues, la elección del tema que se ofrece en estas páginas se debe por un lado, a esa experiencia laboral tan cercana y, por otro, al reconocimiento de una valiosa labor docente realizada, desde hace más de diez años, por un entregado equipo de profesionales comprometidos plenamente con la formación e integración social y laboral de estos alumnos. Hoy, con la experiencia acumulada y dada la temprana aplicación en el IES Duques de Nájera de Logroño de las medidas reguladas por la LOGSE, mediante la impartición de un Programa de Garantía Social Especial (PGSE) de la familia de Artes Gráficas, perfil Imprenta Rápida y manipulados, bien podemos afirmar que este Instituto fue un centro pionero en la Comunidad Autónoma de La Rioja y que apuesta de forma decidida, desde su creación en 1987, por una necesaria atención a la discapacidad ofreciendo una interesante "Respuesta educativa a la diversidad". Para ello se ha abordado una aproximación a los actuales planes de formación que se ofrece a estos alumnos, una vez concluida su etapa escolar obligatoria, recogiendo también la oferta educativa que desde otros centros y colectivos aportan interesantes respuestas de formación e integración para estas personas en la edad adulta. Para el conocimiento de estos recursos ha sido muy valiosa la colaboración de un ex-alumno del centro, Ricardo Zavala Santiago, joven entusiasta que participa activamente en programas europeos y de cuya experiencia y constante formación podremos obtener interesantes conclusiones a lo largo de este trabajo. 
Desde el punto de vista metodológico cabe señalar que se han utilizado diversos métodos de trabajo como la entrevista personal con alumnos, profesores del centro y representantes de entidades y organismos como Inter Europa, junto con la recopilación de información a través de la bibliografía consultada, prensa escrita e internet y otros materiales didácticos que se han considerado de interés para la elaboración de este estudio como folletos informativos, poemas, canciones o películas como Soy Sam o El Milagro de Anne Sullivan. Asimismo, se realizó una "práctica literaria y de pensamiento en el aula" con los alumnos que actualmente están cursando este PGSE en el centro, a través de un sencillo poema ilustrado de Antonio Machado titulado "La plaza tiene una torre", que les permitió reflexionar sobre sí mismos, sus deseos y logros a conseguir en el futuro, elaborando su propio relato a partir de sus ilustraciones sabiendo los alumnos que sus resultados iban a formar parte de este trabajo.

\section{Marco LeGal}

Iniciado el proceso de Transición Democrática en este país, el sistema educativo estaba regulado por la Ley de 1970. Pronto los sucesivos gobiernos de Unión de Centro Democrático (UCD) y PSOE empezaron a plantear la necesidad de una reforma educativa que Ilevaría finalmente a cabo la gestión socialista (1982-1996). Desde el inicio de la reforma, se planteó la necesidad de elaborar una ley que se adaptara no sólo a la nueva realidad educativa, social, cultural y política del país, sino también que abordase, tras amplios tiempos y espacios de debate, las carencias detectadas en el entonces vigente sistema educativo, como la educación de adultos, la educación especial, la respuesta orientadora para los alumnos desde los propios centros, hasta la carga lectiva, materias, sistema de evaluación, promoción, etc. Esta nueva ley recogería, a pesar de sus aciertos y errores, la medida educativa más socializadora de toda la historia educativa de España: la ampliación de dos años de escolaridad gratuita para todos los alumnos, pasando de 14 a 16 años.

La Ley Orgánica de Ordenación General del Sistema Educativo (LOGSE) se aprobó finalmente en 1990, después de largos años de reflexión y debate sobre el nuevo sistema que se quería implantar. Publicada la Ley, ésta necesitó a su vez de un período adicional, no exento tampoco de duros debates y tensiones entre los colectivos implicados, sobre la normativa complementaria que sería necesaria desarrollar para fijar, entre otros, los contenidos de las enseñanzas mínimas en Educación Primaria y Educación Secundaria, o el calendario de una progresiva aplicación de "La Reforma", en aras a conseguir que los jóvenes españoles tuvieran una Enseñanza Obligatoria y gratuita hasta los 16 años de edad, dos años más que los contemplados en la Ley anterior de $1970^{2}$. Se conseguía también con esta medida universalizadora, salvar el vacío legal existente desde la finalización de la edad escolar obligatoria

2. En este sentido son muy ilustrativos los permanentes artículos recogidos en la prensa de la época como los publicados en Comunidad Escolar referidos a "Aprobados cuatro decretos de desarrollo de la LOGSE" y "Función tutorial y orientación educativa", ambos de fecha 19 de junio de 1991. 
establecida hasta entonces en los 14 años, con la edad mínima laboral de 16 años, para poder formalizar un contrato de trabajo.

En este contexto legislativo, los artículos 36 y 37 de la nueva Ley, en su capítulo 5ㅜㅡ, regulaban de manera precisa la Educación Especial, incidiendo en la disposición "de los recursos necesarios para que los alumnos con necesidades educativas especiales, temporales o permanentes, puedan alcanzar, dentro del mismo sistema, los objetivos establecidos con carácter general para todos los alumnos" (artº. 36.1), a la vez que señalaba la necesidad de trabajar de forma inclusiva: "...la atención al alumnado con necesidades educativas especiales, se regirá por los principios de normalización y de integración escolar" (arto․ 36.3). Asimismo, se establecía en el artํ․ 37 la necesidad de que existieran profesores especializados para dar respuesta al alumno desde el momento de su detección, siendo necesario crear los servicios educativos precisos para favorecer su desarrollo y garantizar con ello su escolarización ${ }^{3}$.

En estos quince años de desarrollo de la LOGSE, sí podemos afirmar que se han sentado las bases de una estructura de gestión educativa, con servicios y profesionales especializados en detectar y dar respuesta a los alumnos que presentan necesidades educativas especiales. Las sucesivas transferencias en materia educativa llevadas a cabo en las distintas Comunidades Autónomas del Estado a lo largo de la década de los noventa (en 1999 para La Rioja), han ido favoreciendo la toma de decisiones y medidas que circunscritas a las expectativas planteadas sobre esta materia en cada Comunidad Autónoma tratan de dar solución y respuesta adecuada a una deseable "comprensividad" en la Educación, que estaría íntimamente relacionada "con la dimensión ética, con las ideas y, al mismo tiempo, se proyecta en los fines y estructura de la educación obligatoria de un país, con indudables efectos en la práctica escolar" ${ }^{\prime 4}$.

\section{LOS PROGRAMAS DE GARANTía SOCIAL}

Los Programas de Garantía Social (PGS) constituyen una medida más de atención a la diversidad, con criterios de compensación educativa para los alumnos más desfavorecidos, que no hayan alcanzado los objetivos de la Enseñanza Secundaria Obligatoria y que se encuentren en situación de desventaja sociocultural y laboral. Se pretende ofrecer a los jóvenes que necesitan de un tratamiento educativo alternativo la posibilidad de mejorar sus condiciones para acceder a la vida activa o para poder reinsertarse posteriormente en el sistema educativo reglado.

Esta oferta formativa garantiza su acceso a todos aquellos jóvenes de edades comprendidas entre los 16 y 21 años sin previa titulación de Formación Profesional, lo cual conlleva que los PGS sean susceptibles de recibir un público muy heterogéneo y asumir el dilema de clarificar las actuaciones de orientación psicopedagógi-

3. Ley Orgánica de Ordenación General del Sistema Educativo, Madrid, Ministerio de Educación y Ciencia, 1990.

4. JIMÉNEZ TRENS, María Asunción, El profesorado de la Educación Secundaria ante la diversidad del alumnado en la etapa obligatoria. Colección Digital de Tesis de la UCM: www.ucm.es/ BUCM/2006.htm. 
ca, por un lado y, por otro, buscar una mayor diversificación de modelos para poder dar respuesta positiva a la multiplicidad de necesidades que presentan los jóvenes 5 .

En La Comunidad Autónoma de La Rioja, los estudios de PGS, están claramente etablecidos en la Oferta Educativa que cada año edita la Consejería de Educación y Cultura, en la que se recogen las condiciones necesarias del alumnado para poder cursar estos estudios, así como los centros y programas que se imparten en ellos, la estructura de los mismos, plazos de preinscripción y matrícula ${ }^{6}$. De igual forma se incluyen también los Programas de Garantía Social Especial, orientados a aquellos alumnos con necesidades educativas especiales objeto de este estudio. Los requisitos que deben cumplir los alumnos tanto en la modalidad de Iniciación Profesional como en la de Necesidades Educativas Especiales son:

- Jóvenes menores de 21 años que, al menos, cumplan 16 en el año natural en que inician el programa y no hayan alcanzado los objetivos de la ESO ni posean titulación alguna de Formación Profesional.

- Jóvenes que, cumpliendo los requisitos anteriores, presenten necesidades educativas especiales asociadas a condiciones personales de discapacidad y soliciten acceder a un programa de Garantía Social en la modalidad específica para ellos.

Asimismo, en la modalidad de Iniciación Profesional, podrán matricularse un máximo de 15 alumnos y un mínimo de 10; y en la modalidad para Alumnos con Necesidades Educativas Especiales, el máximo será de 12 y el mínimo de 8 alumnos por grupo. También conviene destacar que los jóvenes con discapacidad pueden matricularse en cualquiera de las modalidades de Garantía Social, pero en este caso conviene orientarles de forma adecuada hacia la opción más integradora de entre todas las posibles.

Los Programas de Garantía Social Especial van dirigidos a alumnos cuyas necesidades educativas especiales están asociadas a condiciones personales de discapacidad, procedentes en su gran mayoría de centros ordinarios. Su duración es de 1.800 horas o dos cursos escolares y se desarrollan en centros educativos públicos y concertados a través de subvenciones a entidades que trabajan con este colectivo de jóvenes y a las cuales nos referiremos más adelante.

Así pues, abordaremos el objeto concreto de nuestro estudio referido a la experiencia docente acumulada, durante los diez últimos años, en la impartición de un Programa de Garantía Social Especial (PGSE) en el IES Duques de Nájera de Logroño, como una interesante respuesta a la diversidad educativa de un alumnado con necesidades educativas especiales, en su mayoría relacionadas con la deficiencia mental. Para ello abordaremos primeramente, una breve reseña referida a la creación del propio centro educativo, su evolución y las enseñanzas que ha ido incorporando

5. AA.VV., De los programas de garantía social a los programas de iniciación profesional: pensamiento del profesorado y del alumnado (Coord. Antonio Sánchez Asín), Barcelona, Laertes-Psicopedagogía, 2004, p. 29-30.

6. Oferta Educativa, curso 2005/2006. Logroño, Consejería de Educación, Cultura y Deporte de la Comunidad Autónoma de La Rioja, 2005. 
desde la implantación de la LOGSE, encaminadas a dar siempre una respuesta educativa a la diversidad de su alumnado. En segundo lugar, abordaremos el PGSE que se imparte en el Centro de Encuadernación básica y manipulados, sus contenidos, estadística del alumnado que lo ha cursado, sus salidas laborales, etc., para concluir con el testimonio del ex-alumno Ricardo Zavala Santiago que ilustra de manera excepcional ese afán de superación constante e integración social y, una "práctica literaria y de pensamiento" Ilevada a cabo con los alumnos que, en el curso 20042005, cursaban estudios en el Centro a través de un poema de Antonio Machado.

\section{La Respuesta educativa a neCesidades específicas en el I.E.S. DuQues de NÁJERA DE LOGROÑo}

\subsection{Creación y evolución del Centro (1987-2005)}

La creación del IES Duques de Nájera se remonta al año 1987 cuya denominación primera fue hasta 1993, Instituto de Formación Profesional $n^{\circ} 2$ de Logroño (BOE no 188 y 246 de 7 de agosto y 14 de octubre de 1987), momento en el que se produce una división del Instituto Politécnico, hoy denominado IES Cosme García, y en el cual se impartían sólo enseñanzas específicas de Formación Profesional de Primer Grado (FP1) de Delineación y de Segundo Grado (FP2), Ramas de Sanitaria: Dietética y Nutrición, Agraria: Viticultura y Enotecnia y Delineación: Edificios y obras, según la Ley General de Educación de 1970.

En el curso 1991-1992, se introdujeron las enseñanzas de R.E.M (Enseñanza Experimental para La Reforma de las Enseñanzas Medias, O.M. 30-09-83), concretamente el primer curso del segundo ciclo de REM, con cuatro grupos. En el curso 1992-1993, se incorporaron en el centro las enseñanzas correspondientes a la LOGSE ( $2^{\circ}$ Ciclo - $3^{\circ}$ ESO) y se mantenía el $2^{\circ}$ curso de REM implantado el año anterior, a la vez que desaparecían paulatinamente los cursos de FP1 y se iniciaba el Módulo Profesional de nivel 2 de Auxiliar de Enfermería, dando paso más tarde a los Ciclos Formativos de Grado Medio y Superior (CFGM y CFGS). De igual forma, en este curso académico se implantaron también los Programas de Integración en ESO para alumnos con necesidades educativas especiales que respondían a la finalidad de integrar este alumnado en el sistema educativo junto con el resto de compañeros atendiendo a su edad y con una ratio de dos alumnos por grupo. Los alumnos de Integración asisten junto con sus compañeros a la mayoría de las asignaturas de $3^{\circ}$ de ESO y salen del aula sólo para determinados ámbitos. Esta respuesta educativa de Integración escolar se impartió en el Centro hasta el curso 1995/1996, fecha del inicio de los Programas de Garantía Social Especial para alumnos con necesidades educativas especiales, en la enseñanza postobligatoria.

Asimismo, desde el año 1993-1994 se imparten también en este Instituto los Programas de Diversificación Curricular para aquellos alumnos que tienen dificultades en el aprendizaje. En un principio estos programas eran de un curso de duración, posteriormente, se fueron ampliando y pasaron a ser de dos cursos académicos ( $3^{\circ}$ y $4^{\circ}$ de Diversificación). Esta respuesta educativa viene ofreciéndose hasta la actualidad y se ha visto complementada, en el curso 1999/2000, con los Programas de Prediversificación para aquellos alumnos que concluido el primer ciclo de ESO, y 
agotados los cursos de repetición, no pueden acceder a $3^{\circ}$ de ESO, por no conseguir los objetivos mínimos de esta etapa educativa. Hasta la fecha este grupo de Prediversificación se ha impartido en el centro con regularidad y a tenor del alumnado que en cada curso académico ha precisado de esta respuesta educativa.

Para concluir, podemos afirmar que desde su creación, en 1987, este centro ha ido transformando las enseñanzas que se impartían como Instituto de Formación Profesional de primer y segundo grado, en un centro educativo donde se imparten en la actualidad enseñanzas de Educación Secundaria Obligatoria (ESO), de Barchillerato de Ciencias de la Naturaleza y de la Salud, de Humanidades y Ciencias Sociales, así como de formación profesional, a través de Ciclos Formativos de Grado Medio y Superior, con la consiguiente respuesta a la diversidad en ESO con grupos de alumnado en Programas de Prediversificación (de un año), Diversificación (de dos años) y PGSE para alumnos con necesidades educativas especiales (de dos años). Es por tanto este IES Duques de Nájera de Logroño un centro que trata de dar cumplida respuesta a los retos educativos que plantea el nuevo siglo, con una diversificada oferta educativa, bien a instancia del propio claustro de su profesorado apostando por una clara y decidida atención a la demanda educativa, social y laboral de su alumnado, como aceptando las propuestas que en cada momento ha ido ofreciendo la propia Administración de cara a abordar una realidad cambiante de intereses sociales que permita dar respuesta tanto a la diversidad del alumnado como a la propia demanda laboral del mismo ${ }^{7}$.

Este esfuerzo integrador se ha reforzado aún más en los últimos años, debido al importante incremento de alumnos procedentes de otros países (Europa del Este, Marruecos, Sudamérica, China), lo que ha puesto de manifiesto la necesidad de conseguir no sólo la integración de este alumnado en sus distintos niveles académicos, homologados por la Administración Educativa atendiendo en su mayoría sólo a criterios de edad, sino también la exigencia de realizar un esfuerzo adicional para conseguir su integración social y de convivencia en el propio centro. Sin duda esta realidad educativa es el auténtico reflejo de lo cambiante de la sociedad actual, que responde en buena medida, a ese constante incremento del flujo de inmigración que de forma continua está caracterizando a la sociedad española y también a esta Comunidad Autónoma de La Rioja y de la que es importante receptora desde mediados de los años noventa.

\subsection{El Programa de Garantía Social Especial: Encuadernación básica y manipulados. Obstáculos para la integración laboral}

Como ya señalamos en el apartado anterior, desde el curso 1992/1993, se implantaron en el centro Programas de Integración en ESO que serían el precedente de los actuales Programas de Garantía Social Especial. Estos últimos vienen impartiéndose a lo largo de diez cursos ininterrumpidos lo que nos permite poder valorar

7. Recientemente se recogía la experiencia educativa del IES Duques de Nájera en la p.11 del Especial de Educación que habitualmente se inserta los miércoles en el periódico La Rioja, de 23 de marzo de 2005, dedicado íntegramente a los Alumnos con necesidades educativas especiales, bajo el titular "Atendiendo a las diferencias". 
su respuesta a la diversidad educativa, desde dos puntos de vista complementarios: por un lado, desde la propia integración de un alumnado con necesidades educativas especiales, en su mayoría referidas a deficiencia mental y, por otro, su posterior seguimiento en cuanto a la inserción de estos alumnos en el ámbito laboral y social.

El perfil del alumnado con necesidades educativas especiales puede describirse como un alumnado que posee en su totalidad el Certificado de Escolaridad y que en buena parte procede del segundo ciclo de ESO, aproximadamente un $30 \%$ de primer curso ( $3^{\circ} \mathrm{ESO}$ ) y un $70 \%$ de segundo curso (4 $\left.\mathrm{ESO}\right)$. En cuanto a las características que presentan los alumnos de este Centro son muy variadas, desde bajo coeficiente de inteligencia con diagnóstico de "retraso mental ligero" hasta deficiencias motoras, Síndrome de Down, etc. Sus edades oscilan entre los 16 y 20 años y su procedencia socio-económica y cultural es muy variada, por lo que las familias de estos alumnos estarían encuadradas desde las de escasos recursos y formación académica, hasta las familias de niveles económicos medios y medios-altos y formación universitaria.

Los fines y metodología de los programas de garantía social especial están orientados a unas adaptaciones curriculares encaminadas a compensar las dificultades de aprendizaje de cada alumno en función de sus capacidades, dentro de su mismo grupo. Para ello se ponen en marcha una serie de mecanismos que tratarán de conseguir la plena integración del alumno estableciendo criterios metodológicos que tengan en cuenta su discapacidad, utilizando técnicas y estrategias para el trabajo individual y en pequeños grupos siendo siempre el alumno el sujeto activo en el proceso de aprendizaje, motivándoles de forma adecuada a partir de sus intereses y capacidades, reforzando positivamente el esfuerzo en el proceso, y no sólo en los resultados.

Para conseguir estos fines es indispensable un apoyo adecuado de medios materiales y humanos, que permita en estos alumnos el favorable desarrollo de sus capacidades desde una perspectiva integradora y normalizadora. El apoyo no sólo debe abarcar la competencia curricular sino también las necesidades de su integración en el Centro o en el grupo (relaciones personales, autonomía, etc.) y su evolución y maduración personal (autoestima, sexualidad, etc.) que englobe todos los ámbitos de su educación. Por todo ello, el apoyo se diseña de forma individual y flexible, siendo complementario el plan de adaptaciones curriculares que se organiza para cada alumno por parte del Departamento de Orientación y más específicamente de los profesores de apoyo del mismo. Igualmente, es importante resaltar que no existe un único modelo de apoyo válido para cualquier alumno y contexto de enseñanza-aprendizaje, sino que el apoyo de un Centro puede ser muy diferente en función de dicho Centro y de su profesorado. Asimismo, el modelo de apoyo estará condicionado por el tipo de necesidades educativas de los alumnos, la metodología y organización en el aula, el tipo de coordinación entre los profesores de apoyo de que se disponga, etc.

El programa de Garantía Social Especial de Imprenta rápida y manipulados que se imparte en el IES Duques de Nájera de Logroño, desde el curso 1995/1996, pertenece a la familia profesional de Artes Gráficas. Durante el curso 2003-2004 estas enseñanzas experimentaron una modificación en las horas lectivas de su programación quedando en la actualidad organizadas en torno a tres áreas de formación. La 
primera, denominada área de formación profesional específica que se distribuye a su vez en dos módulos: el Básico Polivalente y el de Post-impresión de 560 horas cada uno de ellos. La segunda se corresponde con el área de formación básica de 840 horas y la tercera sería el área de formación y orientación laboral de 140 horas ${ }^{8}$

Asimismo, el número de alumnos que han cursado este Programa, en los diez cursos impartidos, atendiendo a su género, es el que se refleja en la siguiente tabla:

ACNEE - CURSOS 1995/96 - 2004/2005

\begin{tabular}{|c|c|c|c|c|c|}
\hline CURSOS & $\mathbf{1}^{\mathbf{o}}$ ENC $\mathbf{M}$ & $\mathbf{1} \mathbf{0}$ ENC $\mathbf{V}$ & $\mathbf{2}^{\mathbf{o}}$ ENC $\mathbf{M}$ & $\mathbf{2} \mathbf{o}$ ENC $\mathbf{~}$ & TOTAL \\
\hline $1995 / 96$ & 1 & 6 & - & - & $7(1 \mathrm{~m}+6 \mathrm{v})$ \\
\hline $1996 / 97$ & 0 & 2 & 1 & 6 & $9(1 \mathrm{~m}+8 \mathrm{v})$ \\
\hline $1997 / 98$ & 1 & 2 & 0 & 2 & $5(1 \mathrm{~m}+4 \mathrm{v})$ \\
\hline $1998 / 99$ & 2 & 3 & 1 & 2 & $8(3 \mathrm{~m}+5 \mathrm{v})$ \\
\hline $1999 / 00$ & 3 & 4 & 2 & 3 & $12(5 \mathrm{~m}+7 \mathrm{v})$ \\
\hline $2000 / 01$ & 1 & 4 & 3 & 3 & $11(4 \mathrm{~m}+7 \mathrm{v})$ \\
\hline $2001 / 02$ & 2 & 1 & 1 & 4 & $8(3 \mathrm{~m}+5 \mathrm{v})$ \\
\hline $2002 / 03$ & 5 & 2 & 1 & 1 & $9(6 \mathrm{~m}+3 \mathrm{v})$ \\
\hline $2003 / 04$ & 3 & 3 & 5 & 2 & $13(8 \mathrm{~m}+5 \mathrm{v})$ \\
\hline $2004 / 05$ & 2 & 3 & 3 & 3 & $11(5 \mathrm{~m}+6 \mathrm{v})$ \\
\hline TOTAL & $\mathbf{2 0}$ & $\mathbf{3 0}$ & $\mathbf{1 7}$ & $\mathbf{2 6}$ & $\mathbf{9 3}(\mathbf{3} \mathbf{m}+\mathbf{5 6} \mathbf{v})$ \\
\hline
\end{tabular}

Como podemos observar de los 93 alumnos matriculados en estos diez cursos, 37 son mujeres y 56 son varones, y la media de alumnos por curso, aunque varía cada año, nunca suele superar los siete alumnos por grupo. De igual forma, partiendo del seguimiento laboral que desde el centro se realiza periódicamente con los alumnos que terminaron dicho Programa, podemos constatar que las dificultades de inserción en el mercado laboral siguen siendo una barrera real para su plena integración social y, aún reconociendo verdaderos logros con excepcionales casos de ocupación en puestos de la Administración o en empresas privadas, sigue siendo mucho lo que aún queda por hacer en este sentido. La sensibilización del empleador hacia este colectivo de personas que a pesar de su discapacidad reúnen, muchos de ellos, las condiciones necesarias para ocupar puestos de trabajo que les permitan una plena integración laboral y social con una equiparación de salarios digna, será el verdadero reto a conseguir en el futuro.

Para ello sería necesario sensibilizar no sólo a los colectivos implicados en la formación de estos alumnos y a sus familias, sino también a la propia Administración

8. El desarrollo y contenido de cada una de estas áreas así como las actividades a realizar en el curso y el plan de trabajo con su horario correspondiente queda claramente especificado en la Hoja Informativa que sobre dicho ciclo elaboró el Orientador del Centro, Ángel Medel Recio y en la información complementaria facilitada por las profesoras de apoyo Pilar López Castillejo y Puy Lasheras Caro a quienes agradezco sus valiosas aclaraciones y aportaciones facilitadas para la realización de este estudio. 
y otros organismos laborales, junto con una necesaria respuesta legislativa que adecuara estos intereses en nuestra Comunidad Autónoma, ya que en esta materia aún está muy lejos de conseguir lo logrado en otras, que incluyen ya en su Oferta de Empleo Público plazas específicas para estas personas. En Logroño podemos contar con el caso excepcional de una alumna que ocupa un puesto de Ayudante de Ujier en el Parlamento de La Rioja, así como el de otros alumnos que trabajan actualmente en algunas empresas privadas, pero a pesar de estos significativos ejemplos el índice de colocaciones es inferior al 50\% de todo el alumnado. De ahí que el gran reto a abordar en un futuro próximo sea el del reconocimiento social de su capacidad para ocupar determinados puestos de trabajo con una justa igualdad de salario que permita hacer realidad su plena integración laboral y social.

\subsection{Las experiencias concretas}

Podríamos destacar aquí muchos ejemplos de superación personal e integración laboral y social, experiencias concretas de alumnos que a lo largo de estos diez cursos académicos fueron pasando por el IES Duques de Nájera de Logroño, como la de una alumna con Síndrome de Down que actualmente ocupa un puesto de Ujier en el Parlamento de La Rioja. También podríamos referirnos a otros muchos alumnos que consiguieron un puesto de trabajo en diferentes empresas de la ciudad y que hoy han visto conseguido su sueño de integración y autonomía personal ${ }^{9}$. Pero he querido centrarme en este apartado dedicado a las experiencias concretas en, Ricardo Zavala Santiago, un ex-alumno que terminó sus estudios en el centro hace nueve años. Mi interés por el seguimiento de este alumno desde su llegada al centro, seguramente estuvo condicionado por la relación de afecto mantenido con sus padres. Aún recuerdo a su madre cuando en los primeros cursos de escolaridad en el Instituto, esperaba paciente la salida de clase de su hijo, siempre leyendo un libro apoyada en la pared del vestíbulo, porque éste aún no tenía autonomía suficiente para ir sólo a casa. Esa paciente espera, con un libro en las manos, auguraba sin duda el resultado de la entrañable entrevista mantenida con este "alumno especial".

Al encontrarnos de nuevo confirmo que su deficiencia mental no le ha impedido durante todos estos años "crecer por dentro" siendo mayor aún si cabe su interés por todo lo que le rodea. Quizá por ello aceptó sin vacilar la propuesta de mantener una entrevista para compartir sus recuerdos, sus proyectos y sus ilusiones. El resultado de la misma, que reproduzco a continuación, es sin duda un testimonio excepcional que he querido incluir en este trabajo porque ilustra lo gratificante que puede llegar a ser una adecuada respuesta a la diversidad educativa "en tiempo y forma" para estos alumnos ${ }^{10}$.

9. La experiencia de integración laboral de la ex-alumna María Otal, Ayudante de Ujier del Parlamento de La Rioja y la de otros alumnos con discapacidad quedó recogida en la prensa local, La Rioja de 11 de mayo de 2002 y de 29 de abril de 2005. También se ofrecen interesantes iniciativas de ámbito nacional en El País, de 5 de febrero de 2005.

10. Otros testimonios de alumnos con necesidades educativas especiales, que también cursaron sus estudios en el centro, y que nos expresan la percepción de su diferencia a través de cartas personales, me han sido facilitados por el Psicólogo y Orientador del IES Duques de Nájera de 


\section{El reto de una reconciliación pendiente con la diversidad de la discapacidad mental}

Ricardo Zavala Santiago tiene hoy 27 años y hace nueve que dejó el IES Duques de Nájera de Logroño para integrarse en la vida adulta. Muestra gran ilusión por esta entrevista pues para él soy una persona cercana, con quien habló en muchas ocasiones en sus cinco cursos de estancia en el Instituto y sabe también de mi amistad con sus padres. Le hace feliz pensar que su experiencia formará parte de un trabajo sobre Respuesta educativa a la diversidad para La Universidad de La Rioja ${ }^{11}$.

Su corpulenta apariencia física se convierte en pura ternura. Me anima a que le pregunte todo lo que yo quiera, "sobre cualquier tema..., trataré de contestar a todo de la mejor manera posible". Al iniciar esta entrevista, me pregunta si soy "además de funcionaria, psicóloga"; su aguda observación me confirma que está muy acostumbrado a reflexionar sobre sí mismo e intuyo que no acaba de comprender muy bien, y con razón, por qué una Licenciada en Historia le ha elegido como protagonista para un trabajo referido a la Diversidad Educativa. Para su tranquilidad le digo que en este discurrir de la vida, todos aprendemos de todos cada día y que personas como él son ejemplos extraordinarios de superación constante. A partir de aquí, trato de que Ricardo evoque sus primeros recuerdos escolares y me explique cuando empezó a ser consciente de su discapacidad mental, a sentirse diferente respecto a sus compañeros.

De sus años de Educación Infantil y Primaria, me refiere que en ese tiempo sus recuerdos son felices, sólo su tristeza quedó fijada en su memoria dos o tres años antes de entrar en el IES Duques de Nájera. Cuando me resume su experiencia, sus recuerdos escolares reflejan todavía en sus palabras "la crueldad" con la que le trataron algunos compañeros de clase y refiere como para él la llegada "al Duques" fue de "total liberación", de alivio, de alegría por el cariño de sus profesores y también por la buena relación entre los compañeros (sus gestos y expresiones refuerzan la nitidez de ese recuerdo, que concibe como la clave de su actitud ante la vida).

Ricardo inició sus estudios en el IES Duques de Nájera en septiembre de 1993, con 16 años de edad. Se incorporó al Programa de Integración dirigido a alumnos de segundo ciclo de Educación Secundaria Obligatoria donde estuvo escolarizado durante tres cursos académicos. Concluida esta etapa, prosiguió sus estudios en el Programa de Garantía Social Especial de encuadernación básica y manipulados y terminó su formación en Junio de 1998.

La primera reflexión que Ricardo hace sobre su llegada al Instituto es la referida a sus profesores. Aún recuerda a todos con sus nombres y apellidos, los cita uno a

Logroño, Ángel Medel Recio y las Profesoras de Apoyo Pilar López Castillejo y Puy Lasheras Caro, a quienes agradezco una vez más su valiosa colaboración.

11. Esta entrevista se realizó el 27 de mayo de 2005, tomando notas, sin grabadora, para que el alumno pudiera expresarse más libremente. A pesar de mi avidez por recoger toda la frescura de sus expresiones, es difícil transmitir las emociones que tanto sus palabras, como sus peculiares gestos o sus silencios aportaron a esta entrevista distendida, en un lugar apacible como el emblemático Café Ibiza de Logroño donde la absoluta tranquilidad del local, a las cinco de la tarde de un día tórrido, fue también importante para que Ricardo Zavala pudiera expresarse sin ruidos ni incomodidades exteriores. 
uno, Ángel Medel, Carmen Villegas, Pilar Calvo, Pilar López y Cristina Castillo. Destaca de todos ellos:

"...el empeño que estos profesores ponían en enseñarnos cosas útiles. En el Instituto la Integración funcionaba muy bien, ante cualquier problema había un grupo de profesores pendientes de nosotros; nos sentíamos muy arropados. Nos explicaron que no teníamos que tener miedo a las notas, que lo importante de la enseñanza no era eso, sino ser capaces de ir solos a pedir lo que necesitábamos a la oficina del colegio, o ir fuera del Centro a la librería de al lado y traer lo necesario. Empecé a disparar para arriba, supe que podía hacer cosas por mí mismo...".

Refiere que el orientador del centro, Ángel Medel, es para él su segundo padre, con quien mantiene todavía, a pesar de los nueve años transcurridos desde su salida del Instituto, una estrecha relación personal. Respecto de las tareas y aprendizaje en los cursos realizados destaca que lo que más le gustaba era encuadernar artesanalmente y todo lo que de este oficio le enseñó su profesora Carmen Villegas. Asimismo, evoca como plastificaban los carnets de los alumnos para la biblioteca del instituto:

"Les hacíamos nosotros, cada alumno del colegio llevaba un carnet hecho por nosotros, y ellos lo sabían. No había rechazo en el cole... yo era el más decidido, me encontraba con gente amable, menos amable y gente que no me entendía. Allí aprendí, en El Duques, que una discapacidad como la mía no era causa de rechazo. Los viernes íbamos de excursión por la ciudad, a conocer Logroño o simplemente a Sabeco... han pasado nueve años y lo recuerdo como si fuera ayer. Tengo 27 años, todo lo que necesite el Duques allá va, allá estoy..."12.

De su etapa posterior a la salida del Instituto, la recuerda como una etapa "más triste", sus distintas experiencias en otros centros como Los Ángeles y Asprodema, no fueron para él tan gratas. Detalla su actual trabajo en esta última, explica cada una de las secciones laborales que comprende este centro y describe con todo detaIle cual es su trabajo concreto haciendo banderillas para la empresa Río Verde. Otros compañeros están en la sección de encurtidos o en la de embotado, pero a él le gusta más hacer banderillas. Su gran inconveniente es que trabaja ocho horas, de 7 de la mañana a 3 de la tarde y cobra unos 300 euros al mes, es consciente de lo injusto del salario y dice convencido "no descarto estar en el futuro en otro lugar"; sabe que éste no será su trabajo definitivo, quiere encontrar un trabajo mejor y con una remuneración más digna.

Me habla de sus proyectos, de su constante formación como Monitor de Tiempo Libre para chicos y chicas con discapacidad, ha realizado cursos de Auxiliar de Lavandería y de Hostelería, pero su mejor experiencia en los últimos años ha sido su participación en el Voluntariado Europeo. De ahí ha surgido la realización de un cortometraje que está elaborando y que piensa presentar en "El Duques", donde quiere contar su estancia de tres semanas en Inglaterra con otros compañeros gra-

12. Su fuerza al referir este sentimiento de gratitud pone de manifiesto su plena disponibilidad para colaborar con el Centro transmitiendo su experiencia a los actuales alumnos que cursan hoy los mismos estudios que él realizó. 
cias a Inter Europa, una asociación dedicada a potenciar el aprendizaje intercultural y la movilidad entre jóvenes con menos oportunidades ${ }^{13}$.

Le invito a que me cuente el contenido de su vídeo y me explica que recoge los testimonios de muchos de los compañeros con quien compartió esa experiencia, procedentes todos de distintos países europeos: "Quiero difundirlo para que vean que las puertas están abiertas para chicos como yo de 18 a 25 años... quiero explicarlo en El Duques...". A lo largo de la entrevista, este ex-alumno repite en varias ocasiones "Quiero seguir para arriba, no sé bien para donde...". También manifiesta sus deseos de encontrar una estabilidad laboral que se adapte "a lo que a mí me gusta, relacionarme con la gente es algo que me encanta". A mi pregunta: ¿Qué te da la gente?, Ricardo hace un significativo silencio, "...me ha ayudado mucho, también me han dado palos duros y también mucho cariño, independencia". Sobre si le gusta salir por la noche, dice: "ahora con el móvil mis padres están más tranquilos, siempre pueden localizarme, pero no me gusta la noche, me aburro, no me gusta nada, prefiero quedarme en casa, leyendo o viendo la tele". En cuanto a sus aficiones señala "me gusta mucho escribir, chatear, escuchar música, viajar, sobre todo desde que estuve en Inglaterra, conocer gente nueva". Le pido que detalle lo que hace una semana cualquiera ya que su padre me advirtió cuando le llamé para concertar esta entrevista: "Ricardo tiene una agenda como la de un ministro". Sonríe asintiendo esa respuesta y relata lo que hace cada día:

"Me levanto a las 6 de la mañana, y a las 6"45 cojo el autobús de la empresa, estoy en mi trabajo de Asprodema de 7 a 15 horas de lunes a viernes, luego por las tardes cada día de la semana tengo una actividad. Los lunes de 6 a 7 voy a la logopeda y a una psicóloga, los martes tengo tutorías del proyecto "Capital Futuro", los miércoles tengo talleres y actividades con Inter Europa, los jueves tutorías y trabajar en el proyecto con el ordenador redactando y preparando..., los viernes también talleres, o simplemente nos vamos a pasear con el grupo de compañeros por Logroño, a disfrutar de la ciudad. Los fines de semana tenemos muchas actividades, campamentos, cine, teatro, excursiones; cada fin de semana es distinto, procuro variar mucho, me gusta hacer cosas siempre diferentes...".

Al referirme a sus padres y lo que éstos suponen para él, sus gestos y sus palabras son bien elocuentes: "a ellos les debo todo". De su padre destaca su constan-

13. El cortometraje realizado por Ricardo Zavala Santiago fue estrenado el día 20 de diciembre de 2005, en el salón de actos de la UNED de Logroño. El periódico La Rioja de 9 de diciembre de 2005, dio amplia información de este proyecto en un interesante reportaje a doble página, en la sección 'Vida y Ocio', elaborado por el periodista Marcelino Izquierdo Vozmediano bajo el titular "Cuando el cine rompe barreras". Asimismo este periódico informaba del estreno de este interesante cortometraje titulado "Ven y así te lo cuento: Experiencias de jóvenes con discapacidad intelectual en el Servicio Voluntariado Europeo" en La Rioja de 21 de diciembre de 2005. De su experiencia en Inglaterra con Inter Europa, la prensa local se hizo eco con un artículo titulado "Fuera las fronteras" en La Rioja de 9 de abril de 2004. Esta misma experiencia se recogería también un año después con otros alumnos en La Rioja, del 19 de febrero de 2005. Asimismo, agradezco a la Asociación Inter Europa la amabilidad con la que atendieron mis consultas, y toda la información facilitada sobre su actividad, especialmente a su directora Alba Moreno por sus valiosas aclaraciones y el interés mostrado en la realización de este trabajo. 
cia, su seriedad: "es un peleón, es el defensor incondicional de mi persona, me encanta mogollón, te mete las cosas en la cabeza... una vez le escuché a mi padre decir que yo nací con discapacidad pero que eso no era impedimento, que tengo otros valores que no tienen los demás". Cuando habla de su madre, su gesto transmite infinita ternura: "es muy, muy, muy cariñosa..., siempre está achuchándome; otras veces en silencio, pero sé que siempre está ahí...". Sin duda las afirmaciones de Ricardo reflejan el agradecimiento infinito a sus padres, sabe que ellos están detrás de todos sus logros, apoyando cada estímulo y cada iniciativa, además del incondicional aval de su amor, de su tesón y su permanente constancia.

Cuando le pregunto sobre su relación con las chicas, me dice "a veces he tenido alguna amiga un poco más especial, pero... es que no tengo tiempo". Sobre sus deseos a corto plazo me habla del vídeo que está realizando y de su interés por encontrar un trabajo relacionado con la atención a las personas con discapacidad "porque es muy humano y sobre todo porque desde que estuve en Inglaterra aprendí muy bien eso de: yo te ayudo ahora a tí porque lo necesitas; tú me ayudas ahora a mí porque lo necesito".

Nos despedimos con el compromiso de seguir estando en contacto a través de internet y le pregunto si me dará permiso para contar en la Universidad de La Rioja todo lo que me ha explicado. Su respuesta fue rotunda: "por supuesto que sí... al Duques le debo todo". En la despedida, también le digo que ha sido un verdadero regalo haber podido compartir sus recuerdos, sus logros y sus deseos; que hacen falta personas como él para reconciliarnos con la diversidad de la discapacidad y siento que este alumno hubiera sido uno de los preferidos de Ortega por el estricto cumplimiento de sus consejos:

"Sed entusiastas, verted a manos llenas y en toda pureza vuestra vida...

El amor a todas las cosas es el entusiasmo..., cultivad en vosotros este hábito de transmigrar, de trasladar vuestro ánimo a cuanto os rodea"14.

\section{Propuesta de una "práctica literaria y de pensamiento" en el aula, A PARTIR DE UN POEMA DE ANTONIO MACHADO ${ }^{15}$}

Para cerrar esta reflexión sobre la respuesta educativa a la diversidad, asociada a deficiencias mentales consideré que para unir el pasado y el presente de esta pro-

14. ORTEGA Y GASSET, José, "Elogio de las virtudes de la mocedad". Revista de Occidente, no 15-16, Extraordinario III (1982), pp. 65-71. (Debo el conocimiento de este texto al profesor de Filosofía del IES Duques de Nájera de Logroño, Rafael Garlito Rosado, a quien agradezco su valiosa aportación por ilustrar magníficamente la personalidad de Ricardo Zavala Santiago). Después del visionado en el aula de la película Yo soy Sam, propuesta por la profesora Dra. D ${ }^{a}$. M ${ }^{a}$ Asunción Jiménez Trens en el curso de doctorado, la arrolladora personalidad de Ricardo es muy identificable con la del protagonista del film, interpretado excepcionalmente por el actor Sean Penn.

15. De la realización paralela de este curso de doctorado sobre "Respuesta educativa a la diversidad" con el impartido por la Dra. Da María José Clavo Sebastián sobre El problema del hombre en el pensamiento filosófico español de finales del siglo XIX y de mi exposición en éste sobre "La filosofía de Antonio Machado en sus poetas apócrifos Abel Martín y Juan de Mairena" surgió la idea de plantear esta "práctica literaria y de pensamiento" con los alumnos en el aula. 
puesta educativa, debía enlazar el testimonio de este ex-alumno singular, Ricardo Zavala Santiago, integrado ya en la vida adulta, con el acercamiento a los actuales alumnos del centro, los cuales todavía adolescentes disponen hoy de un importante tiempo de formación en el mismo, tratando con ello de aproximarnos de manera más directa a su actual realidad educativa.

El hilo conductor que iba a hacer posible mi propuesta de trabajo con los alumnos que cursan este Programa en el Centro (cuatro alumnos de primer curso y seis alumnos de segundo curso) consistió en tratar de interiorizar un breve poema de Antonio Machado titulado "La Plaza tiene una torre", a partir de una sugerente edición ilustrada por Jesús Gabán para una colección infantil, como interesante material a trabajar con ellos dada la sencillez del mismo y sus atractivas ilustraciones:

$$
\begin{aligned}
& \text { La plaza tiene una torre } \\
& \text { la torre tiene un balcón } \\
& \text { el balcón tiene una dama } \\
& \text { la dama una blanca flor } \\
& \text { ha pasado un caballero } \\
& \text { iquién sabe por qué pasó! } \\
& \text { y se ha llevado la plaza } \\
& \text { con su torre y su balcón } \\
& \text { con su balcón y su dama } \\
& \text { su dama y su blanca flor }{ }^{16} \text {. }
\end{aligned}
$$

Mi intención inicial fue captar su interés mediante la propia colocación en el aula de los alumnos a modo de tertulia distendida y con el empleo de distintas modulaciones de voz para cada uno de los versos leídos, a fin de conseguir su total atención, incluyendo brevísimos silencios ante las observaciones realizadas y mi permanente mirada sobre todos ellos (esa "mirada faro" de la que nos habló el pro-

16. La plaza tiene una torre, Madrid, Alhambra Longman, 1991. (Ilustraciones realizadas por Jesús Gabán, para el poema homónimo de Antonio Machado). Es obligado referir aquí que sin la colaboración de Ángel Medel Recio, Psicólogo y Orientador del IES Duques de Nájera al aceptar sin vacilación mi propuesta de realizar en el aula una "práctica literaria y de pensamiento", esta actividad no hubiera existido; tampoco se hubiera podido plantear tal y como la diseñé sin su fundamental colaboración con los recursos informáticos encargándose de reproducir, en una sesión previa con los alumnos, las ilustraciones del poema objeto de esta práctica. También sin su pericia como internauta no hubiera podido concluir esta actividad localizando en la red la emblemática canción de Víctor Manuel "Sólo pienso en ti" elegida para cerrar mi intervención, a modo de homenaje al amor surgido entre dos alumnos de clase. Su audición nos permitió no sólo escuchar esta bellísima canción sobre el amor de una pareja con discapacidad mental -tan familiar para mi generación pero totalmente desconocida para los alumnos- sino también reflexionar sobre el origen de la discapacidad de sus protagonistas desde el momento mismo de su nacimiento y cómo, en la edad adulta, surgió su enamoramiento en el comedor del internado donde ambos se encontraban. Asimismo, sin la decisiva colaboración de Pilar López Castillejo, profesora de apoyo, esta práctica tampoco hubiera logrado los interesantes resultados literarios que se consiguieron ya que fue fundamental su labor posterior en el "taller de redacción" trabajando con los alumnos la propuesta formulada de elaborar su propio relato sobre lo que les sugería el poema de Antonio Machado a partir de las reflexiones hechas en el aula. 
fesor Fermín Navaridas como importante recurso a emplear en el aula para captar la atención de todo el alumnado) ${ }^{17}$.

Los primeros minutos estuvieron dedicados a mi presentación, a explicar el motivo por el cual estaba en el aula, a señalar el tema que íbamos a abordar y a despertar su deseo de participar, al objeto de que los alumnos se sintieran verdaderos protagonistas de la actividad. En una segunda fase, escucharon atentamente la lectura que les hice del poema seleccionado, marcando distintas modulaciones de voz para atraer su interés, después memorizamos todos juntos cada verso a través de las ilustraciones y a continuación, dos alumnos voluntarios leyeron a sus compañeros cada una de las dos estrofas del poema tratando de modular también sus voces para transmitir mejor el significado de cada verso. Posteriormente, les indiqué sobre cada ilustración las diferentes lecturas que podíamos hacer del poema:

- Una más sencilla, la que todos vemos, la bella dama y el apuesto caballero que se encuentran y se van enamorados y felices 'comiendo perdices'.

- Otra más compleja, sería la que nos proporciona una mirada más atenta, más reflexiva, tratando de buscar otros significados en las palabras:

- la plaza, la torre, el balcón y la dama pueden representar nuestras seguridades; mi país, mi ciudad, mi casa, mi entorno, mi yo, la seguridad de lo conocido, mi torre.

- La joven que se asoma al balcón con sus ilusiones, sus proyectos y sus sueños acariciados en la rosa blanca podemos ser nosotros mismos, con nuestros deseos, nuestros proyectos para el futuro, para cuando ya no estemos en el Instituto.

- El apuesto caballero que pasa y recoge todo, la plaza, la torre, el balcón y la dama, quizás simbolice también los días tristes, nuestras inseguridades, esa pérdida de ilusiones... que el caballero se va a llevar en su mano; pues en el discurrir de la vida, tanto los jóvenes como los adultos, unos con una discapacidad y los demás, seguramente con otras, todos tenemos días en que creemos ser muy felices y otros que por el contrario, nos sentimos más tristes, más solos, decepcionados porque nuestros deseos no se cumplen como esperábamos ${ }^{18}$.

17. El desarrollo de la clase cuya duración aproximada fue de unos 40 minutos, se impartió el día 16 de junio de 2005, en la segunda hora lectiva de la mañana, y estuvo dividida en varias fases de apenas siete $u$ ocho minutos cada una de ellas.

18. Recientemente la periodista y escritora Rosa Montero nos presentaba en "Maneras de Vivir: Elogio del malestar" su particular mirada sobre esta misma reflexión en El País Semanal de 5 de junio de 2005, p. 138. Asimismo el egregio filósofo Emilio Lledó, en su reciente obra Elogio de la infelicidad, Valladolid, Cuatro Ediciones, 2005, p. 7, sostiene que "la felicidad... es una idea casi trivial y, a la vez, una aspiración insoslayable, medio perdida en 'ese horizonte de sueños, ideales, deseos, utopías, amenazas y dolores' que nos atrapa y nos envuelve. Pero si, dadas las múltiples contiendas de la vida, parece querer imponerse siempre entre nosotros la infelicidad, conviene sin embargo reconocerla, usarla y esquivarla, luchar contra su fatalidad en la medida de nuestras limitaciones, reconocer sus frutos y convertirla en una palanca para tratar con nosotros mismos". 

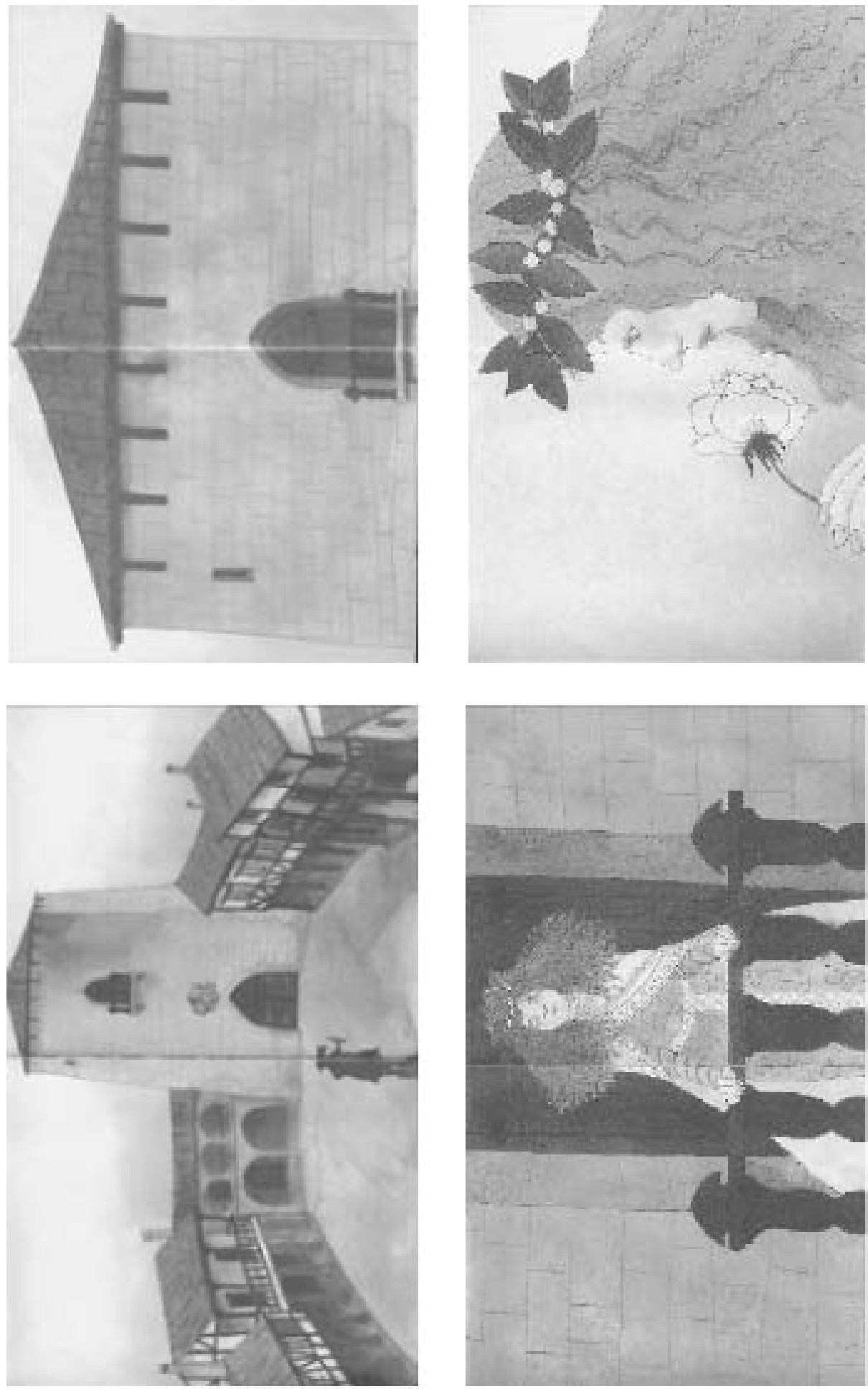


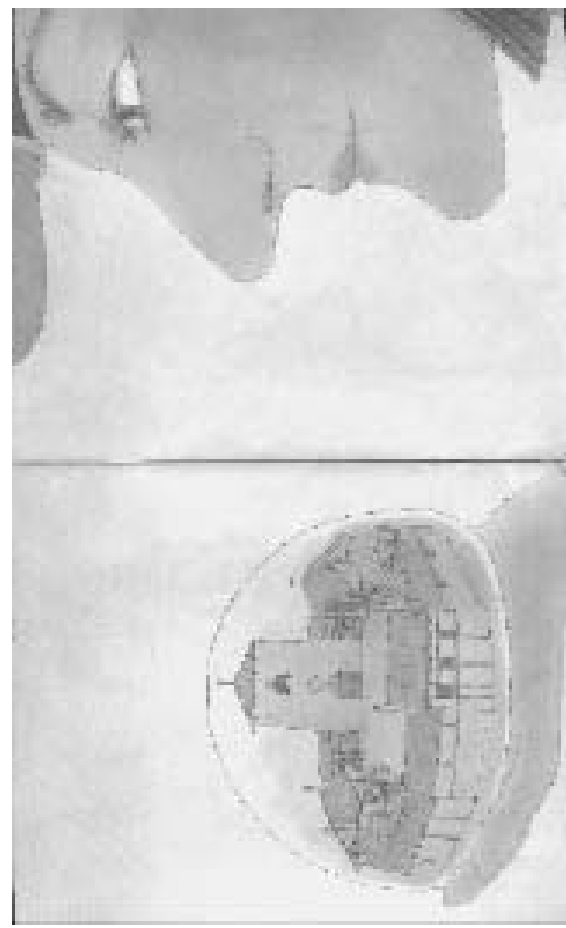

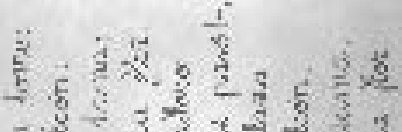

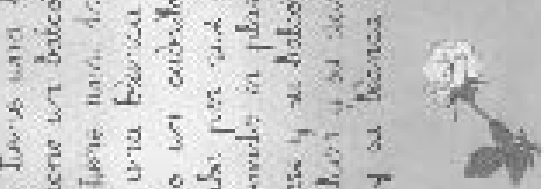

$$
\begin{aligned}
& \text { क } \\
& 3 \text { y }
\end{aligned}
$$

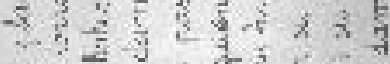

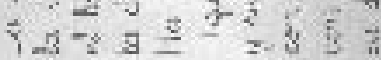
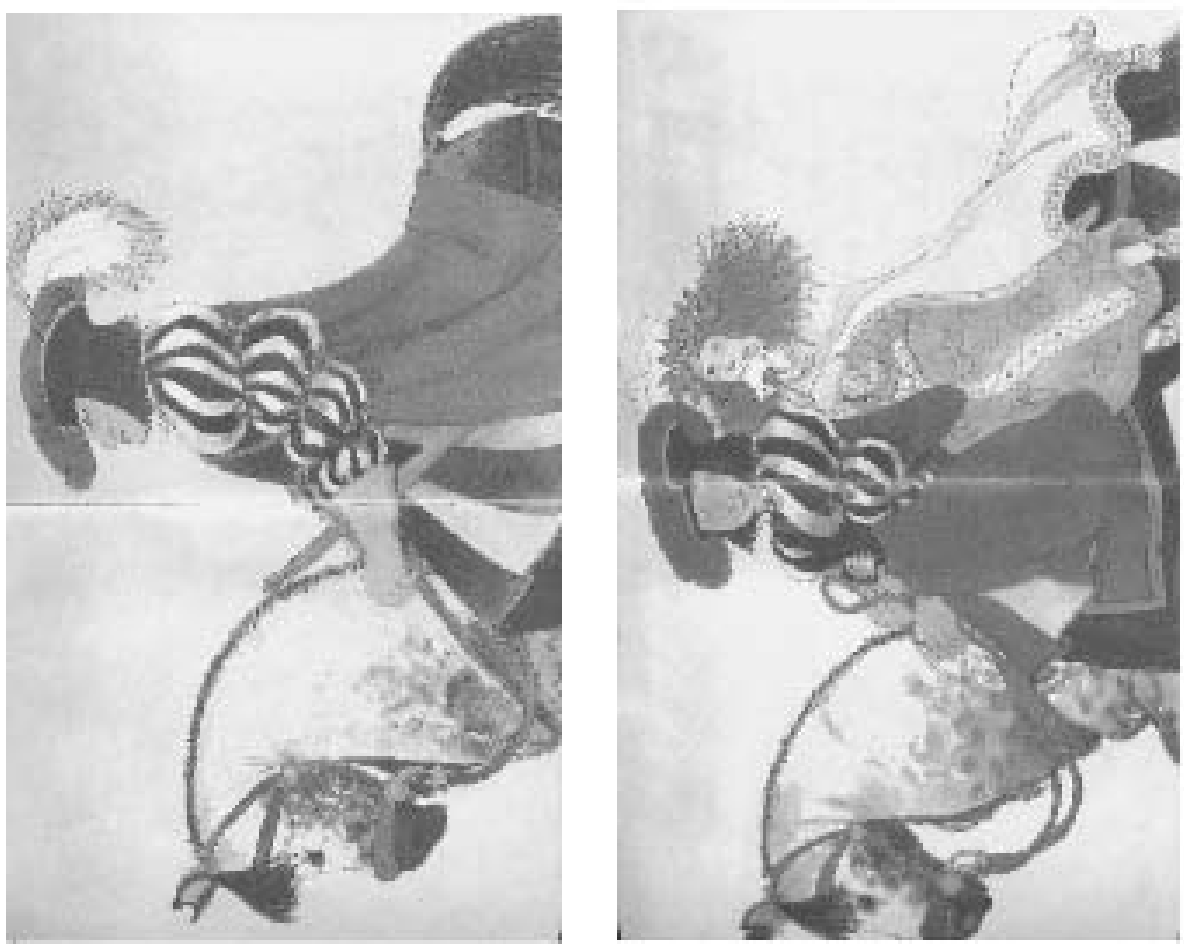
Tendremos entonces que empezar de nuevo; refugiarnos si es necesario en un poema como éste o en una bella canción, o en las personas que de verdad queremos, para ser más fuertes, para no dejarnos vencer...; para recuperar la alegría, la sonrisa, la fuerza que nos permita seguir adelante sin olvidar que, aún con nuestras discapacidades, tenemos la suerte de haber nacido en este llamado primer mundo, donde hay escuelas que nos acogen para atender nuestra discapacidad y medios para ayudarnos a ser más felices. Tampoco debiéramos olvidar que en otros lugares del planeta hay muchos otros adolescentes que lo tienen más difícil, ejemplos como el del pueblo saharaui viviendo desde hace tres décadas en campos de refugiados en pleno desierto, o el de países de extrema pobreza y en constantes guerras, deben hacernos reflexionar y ser un estímulo de superación constante. Sugerencias todas ellas encaminadas a despertar su capacidad de reflexión sobre el mundo en el que viven y sobre sí mismos ${ }^{19}$.

Después se les entregó un folio con las imágenes del poema ilustrado de Antonio Machado, las cuales ya les eran familiares por haberlas escaneado en sesiones anteriores con su profesor en el aula de informática. Asimismo, junto con las imágenes se les adjuntó una propuesta de trabajo donde debían responder al siguiente planteamiento:

"El poema que escribió Antonio Machado "La Plaza tiene una torre" nos ha sugerido hoy muchas ideas.

Observa atentamente las ilustraciones y escribe lo que este poema te sugiere.

Redacta tu propio relato con estos personajes y explica cuales son tus sueños y tus ilusiones para el futuro".

Es curioso constatar al analizar los resultados obtenidos, cómo algunos alumnos interiorizaron las dos propuestas sugeridas sobre el poema, mezclando todas las reflexiones; de modo que la mayoría quería encontrar un príncipe o princesa con quien casarse y tener hijos y ser muy felices en "un chalet en la playa" o en "un piso con piscina"; mientras que otros ofrecían reflexiones en las que nos hablaban de sí mismos y de sus sentimientos, como la identificación que hacía una alumna de la doncella del poema con "...mi amiga Verónica que cogió la flor blanca desde el cielo". También quedó evidenciado, en sus respectivas redacciones, el diferente grado de discapacidad mental, bien a través de la madurez de su caligrafía manifestada a su vez en el empleo de lápiz o bolígrafo, o por el lenguaje empleado en la composición de su redacción y el contenido de sus reflexiones:

19. En este sentido podría realizarse otra práctica complementaria en el aula con la proyección del documental El viaje de Susu del director Nicolás Muñoz, que capta esa ida y vuelta de un niño entre el campamento de refugiados saharauis de Smara, en un desierto de piedras junto a la ciudad argelina de Tinduf, y su experiencia cuando vienen a España en el verano. Otra extraordinaria película que ilustra muy bien la capacidad de adaptación de los niños al entorno, por muy crudo que éste sea, concretamente en un campo de refugiados kurdos es Las tortugas también vuelan. Sobre la valoración de estos testimonios en el reportaje titulado "Susu, del Sáhara a Madrid", en El País Semanal de 22 de mayo de 2005, pp. 20-29. 
"Espero ser feliz, y que me vaya bien, espero que no haya guerra y pobreza ni rechazo a las demás razas y ser todos felices y ayudarnos entre todos, que no se enfrenten ni que se peleen, que primero hablen antes de pelearse y ser felices todos juntos".

Por el contrario, otra alumna elaboraba un original final para el poema y para sus sueños de futuro:

"...viene un príncipe, tiene cara de enfadado, coge la plaza con la torre, coge a la dama con su flor, le va a dejar en el bosque a la dama, él se va y la deja abandonada. Me gustaría ser bailarina de sevillanas en el futuro".

Para concluir mi intervención seleccioné el tema musical de Víctor Manuel titulado "Sólo pienso en ti" que escuchamos a través del ordenador pudiendo no sólo compartir su melodía, sino también, la hermosa historia de amor surgida entre sus protagonistas y el origen de su discapacidad mental.

Sinceramente, considero que el objetivo planteado con los alumnos a través de esta sencilla "práctica literaria y de pensamiento" fue ampliamente cumplido, siendo muy gratificante la experiencia vivida en el aula, donde pude constatar también el permanente interés del alumnado por el poema de Antonio Machado y cada una de las reflexiones planteadas en cada ilustración y cada verso, siendo mayor mi asombro ante su reiterada petición de seguir escuchando esa bella canción de Víctor Manuel, hasta entonces inédita para ellos.

\section{CONCLUSIONES}

Una vez abordada la contextualización previa en el orden legislativo sobre la regulación de las enseñanzas para alumnos con discapacidad mental a partir de la Logse y la detenida valoración sobre los Programas de Garantía Social contemplados en ella, tanto de Iniciación Profesional como de Educación Especial, nos hemos centrado en la implantación y transformación de las enseñanzas impartidas en el IES Duques de Nájera de Logroño, desde su creación en 1987 hasta la actualidad, como "centro pionero" en esta Comunidad Autónoma de La Rioja en ofrecer respuestas educativas a la diversidad entre las que cabe señalar su experiencia docente a través del Programa de Garantía Social Especial: Imprenta Rápida y Manipulados. Tras la presentación del contenido específico del mismo, sus materias, carga lectiva, estadística del alumnado, expectativas de empleo, etc.; sólo cabía incluir experiencias personales de los alumnos que nos hablaran del pasado y el presente de esta interesante y fructífera respuesta educativa en sus diez años de desarrollo.

A modo de conclusión podemos afirmar que es constatable como la respuesta educativa a la diversidad se hace cada día más necesaria en las aulas y las escuelas de nuestro país, no sólo por el vertiginoso cambio social al que estamos asistiendo, con los permanentes flujos de inmigración de los más variados países (Europa del Este, Latinoamérica, China, India, Pakistán, Marruecos...), sino también por la propia transformación de la familia española, donde "parejas de hecho, bebés fuera del matrimonio y hogares monoparentales marcan el cambio" de los tradicionales mode- 
los familiares dando lugar a una real multiplicidad de formas de convivencia, que están teniendo su vivo reflejo, también en los centros escolares ${ }^{20}$.

En cuanto a las peculiares aptitudes del alumnado debemos de entender la diversidad como el hecho que diferencia a cada individuo, en el sentido de que "todos somos diversos", ¿quién no necesitó apoyo en un momento determinado de la vida? ¿qué alumno necesita hoy más atención en el aula, el que procede de otro país, o el que está viviendo la separación de sus padres? ¿el que tiene una discapacidad mental, o el superdotado?, por poner tan sólo algunos ejemplos.

Respecto a la Educación Especial, cabe señalar que los avances en cuanto a respuestas educativas han sido muy significativos en España desde la aprobación de la LOGSE en 1990, pero sigue siendo un reto el conseguir la plena integración social y laboral de las personas con discapacidad, dada la falta de sensibilidad por parte de la propia sociedad y la importante dificultad de conseguir un empleo en la edad adulta, con garantía de un salario digno.

También es cierto que si en el ámbito educativo hay una mayor "conciencia del diferente" como "valor que nos enriquece", no es menos cierto que todavía quedan muchas iniciativas por abordar, tanto de orden legislativo, como empresarial, social, escolar y familiar, para que la integración de las personas con discapacidad mental sea un hecho real en nuestra sociedad. Entretanto, quizá debiéramos repasar la sabia enseñanza de Antonio Machado a través de su maestro apócrifo Juan de Mairena:

"Yo os enseño en fin o pretendo enseñaros... el amor al prójimo y al distante, al semejante $y$ al diferente, y un amor que exceda un poco al que os profesáis a vosotros mismos, que pudiera ser insuficiente"21.

\section{Bibliografía}

APARISI I ROMERO, J. A. (1998), "EI marco legislativo de los Programas de Garantía Social en la Comunidad Valenciana: Posibilidades y límites". En MARHUENDA FLUIXÁ, F. y MARTíNEZ MORALES, I. (coord.) (1998), Experiencia educativa de los Programas de Garantía Social: Actas de las jornadas de reflexión y debate sobre los Programas de Garantía Social en las políticas de inserción sociolaboral en la Comunidad Valenciana, Colegio Mayor Universitario La Coma, 3 y 4 de abril de 1998. Valencia: Servicio de Publicaciones de la Universitat de València, pp. 19-34. ARROYO ALMARAZ, A., CASTELO MORENO, A. y PUEYO CAUDEVILLA, Ma C. (1991). El Departamento de Orientación: Atención a la diversidad [Orientación y tutoría: materiales 12-16 para Educación Secundaria]. Madrid: Narcea, MEC. BRIOSO, A. y SARRIÁ, E. (1991). "Transtornos de comportamiento". En PALACIOS, J., MARCHESI, A. y COLL, C. (comp.) (1991). Desarrollo psicológico y educa-

20. En este sentido es muy esclarecedor el exhaustivo análisis ofrecido en El País, 19 de junio de 2005 con motivo de la inminente aprobación del matrimonio homosexual bajo el titular "La transformación de la familia española", p. 44.

21. CEREZO GALÁN, P. Palabra en el tiempo. Poesía y filosofía en Antonio Machado, Madrid, Gredos, 1975, p. 600. 
ción, III. Necesidades educativas especiales y aprendizaje escolar. Madrid: Alianza, pp. 183-196.

COBO CATENA, B. (1999). "El huerto escolar en los programas de garantía social". En RUBIO, E. y RAYÓN, L. (coord.). Repensar la enseñanza desde la diversidad. Sevilla: Publicaciones M.C.E.P., Kikirikí Cooperación Educativa, pp. 131-138.

ECHEITA, G. y MARTíN, E. (1991). "Interacción social y aprendizaje". En PALACIOS, J., MARCHESI, A. y COLL, C. (comp.) (1991). Desarrollo psicológico y educación, III. Necesidades educativas especiales y aprendizaje escolar. Madrid: Alianza, pp. 49-67.

EQUIPO DE INVESTIGACIÓN ESCUELA TRABAJO (2002). "Prospectiva en torno a las grandes dimensiones de los Programas de Garantía Social". Revista de educación, 329, 487-512.

FIERRO, A. (1991). "La escuela frente al déficit intelectual". En PALACIOS, J., MARCHESI, A. y COLL, C. (comp.) (1991). Desarrollo psicológico y educación, III. Necesidades educativas especiales y aprendizaje escolar. Madrid: Alianza, pp. 277-290.

FIERRO, A. (1991). "Los niños con retraso mental". En PALACIOS, J., MARCHESI, A. y COLL, C. (comp.) (1991). Desarrollo psicológico y educación, III. Necesidades educativas especiales y aprendizaje escolar. Madrid: Alianza, pp. 267-275.

GRUP D'INVESTIGACIÓ ESCOLA TREBALL (2004). "Conocer y mejorar las prácticas docentes en los programas de garantía social". Herramientas: revista de formación y empleo, 75, 36-42.

HUESO VILLEGAS, Maㅡㄹ D. y GARCÍA MOLINA, I. (1997). "Los programas de garantía social y los jóvenes con necesidades educativas especiales en la comunidad autónoma andaluza". En TORRES GONZÁLEZ, J. A. (coord.). La innovación de la educación especial (actas de las XIV Jornadas Nacionales de Universidad y Educación Especial). Jaén: Universidad de Jaén, pp. 223-232.

JIMÉNEZ TRENS, M. A. (2003). El profesorado de la Educación Secundaria ante la diversidad del alumnado en la etapa obligatoria. Tesis Doctoral. Madrid: Universidad Complutense de Madrid. Disponible en: http://www.ucm.es/BUCM/tesis/ edu/ucm-t26875.pdf [última consulta: 22/03/2006].

LEÓN GUERRERO, Mํ. J. (2000). "Los programas de garantía social: una propuesta de formación sociolaboral". En MIÑAMBRES ABAD, A. y JOVÉ MONCLÚS, G. (coord.). Atención a las necesidades educativas especiales de la Educación Infantil a la Universidad (actas de las XVII Jornadas Nacionales de Universidad y Educación Especial). Lleida: Universidad de Lleida, pp. 249-260.

MARHUENDA FLUIXÁ, F. (1998). "La organización de las enseñanzas en los Programas de Garantía Social". En MARHUENDA FLUIXÁ, F. y MARTíNEZ MORALES, I. (coord.) (1998), Experiencia educativa de los Programas de Garantía Social: Actas de las jornadas de reflexión y debate sobre los Programas de Garantía Social en las políticas de inserción sociolaboral en la Comunidad Valenciana, Colegio Mayor Universitario La Coma, 3 y 4 de abril de 1998. Valencia: Servicio de Publicaciones de la Universitat de València, pp. 97-122.

MARTÍNEZ JIMÉNEZ, J. (1999): "Descripción y análisis de una experiencia de garantía social". Contextos Educativos: Revista de educación, 2, 229-256. 
MARTÍNEZ LÓPEZ, R. (1998), "Las tareas de enseñanza en los Programas de Garantía Social". En MARHUENDA FLUIXÁ, F. y MARTíNEZ MORALES, I. (coord.) (1998), Experiencia educativa de los Programas de Garantía Social: Actas de las jornadas de reflexión y debate sobre los Programas de Garantía Social en las políticas de inserción sociolaboral en la Comunidad Valenciana, Colegio Mayor Universitario La Coma, 3 y 4 de abril de 1998. Valencia: Servicio de Publicaciones de la Universitat de València, pp. 203-21.

MARTÍNEZ LÓPEZ, R. y MONTOLIU FERRERA, B. (1998), "La evaluación en los Programas de Garantía Social". En MARHUENDA FLUIXÁ, F. y MARTíNEZ MORALES, I. (coord.) (1998), Experiencia educativa de los Programas de Garantía Social: Actas de las jornadas de reflexión y debate sobre los Programas de Garantía Social en las políticas de inserción sociolaboral en la Comunidad Valenciana, Colegio Mayor Universitario La Coma, 3 y 4 de abril de 1998. Valencia: Servicio de Publicaciones de la Universitat de València, pp. 213-220.

PALACIOS, J., MARCHESI, A. y COLL, C. (comp.) (1991). Desarrollo psicológico y educación, III. Necesidades educativas especiales y aprendizaje escolar. Madrid: Alianza.

RODRÍGUEZ GALLO, J. L. (2003), "Los programas de garantía social para alumnos con necesidades educativas especiales como formación práctica para el empleo". En CASADO MUÑOZ, R. y CIFUENTES GARCÍA, A. (coord.). El acceso al empleo y a la universidad de personas con discapacidad : barreras y alternativas (actas de las III Jornadas sobre Universidad y Personas con Discapacidad, I Reunión Científica sobre la Repuesta Socioeducativa a la Discapacidad). Burgos: Servicio de Publicaciones, Universidad de Burgos, pp. 271-278.

ROGERO ANAYA, J. y GORDO, J. L. (2000), "Programas de garantía social y aulastaller: entre el deseo y la realidad". Cuadernos de Pedagogía, 293, 48-52.

RUIZ SERRA, R. (1998), “ ¿Hacia dónde se dirigen los Programas de Garantía Social? Objetivos de los programas de Garantía Social desde una triple perspectiva”. En MARHUENDA FLUIXÁ, F. y MARTÍNEZ MORALES, I. (coord.) (1998), Experiencia educativa de los Programas de Garantía Social: Actas de las jornadas de reflexión y debate sobre los Programas de Garantía Social en las políticas de inserción sociolaboral en la Comunidad Valenciana, Colegio Mayor Universitario La Coma, 3 y 4 de abril de 1998. Valencia: Servicio de Publicaciones de la Universitat de València, pp. 197-202.

SÁNCHEZ ASÍN, A. (coord.) (2004). De los programas de garantía social a los programas de iniciación profesional: pensamiento del profesorado y del alumnado. Barcelona : Laertes.

VILLAR EPIFANIO, V. (2003), "Discapacidad: La Diversidad como fuente del enriquecimiento social". En CASADO MUÑOZ, R. y CIFUENTES GARCÍA, A. (coord.). EI acceso al empleo y a la universidad de personas con discapacidad : barreras y alternativas (actas de las III Jornadas sobre Universidad y Personas con Discapacidad, I Reunión Científica sobre la Repuesta Socioeducativa a la Discapacidad). Burgos, Servicio de Publicaciones, Universidad de Burgos, 279-288.

ZACARÉS GONZÁLEZ, J. J. (1998), "Características psicosociales de los jóvenes participantes en los programas de garantía social: una aproximación a partir de la 
metodología de cuestionario". En MARHUENDA FLUIXÁ, F. y MARTíNEZ MORALES, I. (coord.) (1998), Experiencia educativa de los Programas de Garantía Social: Actas de las jornadas de reflexión y debate sobre los Programas de Garantía Social en las políticas de inserción sociolaboral en la Comunidad Valenciana, Colegio Mayor Universitario La Coma, 3 y 4 de abril de 1998. Valencia: Servicio de Publicaciones de la Universitat de València, pp. 35-66.

\section{Canción/Videoclip}

Tema musical compuesto por Víctor Manuel San José: "Sólo pienso en ti" (en Mucho más que dos, 1994 y Vivir para cantarlo, 1999).

\section{Filmografía}

El milagro de Anne Sullivan (1962). Director: Arthur Penn.

Yo soy Sam (2001). Directora: Jessie Nelson.

\section{Láminas}

La plaza tiene una torre, Madrid, Alhambra Longman, 1991. (Ilustraciones realizadas por Jesús Gabán, para el poema homónimo de Antonio Machado). 